\title{
Enhanced Mechanical Properties of Polyurethane Composite Coatings through Nanosilica Addition
}

\author{
Suraj Maganty ${ }^{1}$, Maria P. C. Roma ${ }^{1}$, Stephan J. Meschter ${ }^{2}$, Dale Starkey ${ }^{3}$, Mario Gomez ${ }^{3}$, David \\ G. Edwards ${ }^{3}$, Abdullah Ekin ${ }^{4}$, Kevin Elsken ${ }^{4}$, and Junghyun Cho ${ }^{1, *}$ \\ ${ }^{1}$ Binghamton University (SUNY), Department of Mechanical Engineering \& Materials Science \\ and Engineering Program, Binghamton, NY 13902 \\ ${ }^{2}$ BAE Systems, Electronics Systems, Endicott, NY 13760 \\ ${ }^{3}$ Henkel Electronic Materials LLC, Irvine, CA 92606 \\ ${ }^{4}$ Bayer MaterialScience LLC, Pittsburgh, PA 15205
}

\begin{abstract}
Tin rich surfaces inside $\mathrm{Pb}$-free electronics are more prone to tin whisker growth, thereby resulting in electrical failure of components. Conformal polyurethane (PU) coatings, which can be used as a mitigation strategy for such whisker growth, were investigated in this study. Nanoparticles of silica ( $20 \mathrm{~nm}$ in diameter) were added via liquid suspension to the PU resin to improve its mechanical properties. Four compositions of functionalized nanosilica suspensions, 10 wt.\%, 20 wt.\%, 30 wt.\% and 50 wt.\% (corresponding to the solid silica weight of $3.50 \%$, $6.74 \%, 9.75 \%$, and $15.17 \%$, respectively) were evaluated to establish the relationship between microstructure developments and mechanical properties. Fourier-transform infrared spectroscopy (FTIR) and low-kV field emission scanning electron microscopy (FE-SEM) were used to examine the effects of nanosilica suspension addition to the PU resin. Mechanical properties of the coating were not only evaluated by nanoindentation for localized PU matrix properties but also by uniaxial tensile testing for composite properties. Results from both scales show that the addition of the particle suspension up to $20 \mathrm{wt} . \%$ (corresponding to $9.75 \mathrm{wt} . \%$ nanosilica) makes optimum mechanical performance consisting of good modulus, strength and ductility. This conclusion is also supported from microstructural, as well as chemical aspects of the PU coatings with the addition of nanosilica suspension.
\end{abstract}

[keywords: conformal; coatings; polyurethane; nanosilica; Pb-free; tin whisker]

\footnotetext{
*Corresponding author: jcho@binghamton.edu 


\section{Introduction}

Electronics components and boards which utilize pure tin, tin-rich surfaces and tin-based solders have become more prevalently used these days following the Restriction of Hazardous Substances (RoHS) directives banning the use of lead $(\mathrm{Pb})$ [1]. One issue associated with the increased usage of tin is the appearance of tin 'whiskers' which nucleate and grow from the stressed tin-rich surfaces resulting in metallized surface damage and unwanted debris inside the package, cause current leakage, and may often cause catastrophic failure. Polymer systems such as commercially available parylene-C and other generic polyurethanes (e.g., Arathane 5750 ${ }^{\mathrm{TM}}$ ) have been considered for being used in whisker mitigation coating strategies $[2,3]$. Nevertheless, these coating techniques tend to be cost prohibitive (in case of parylene-C) and often involve complex processing [4]. A coating technique which is economical and requires relatively simple process parameters is thus desired. In addition, reworkability is another important criterion for these conformal coatings.

Polyurethanes are segmented block copolymers that inherently possess excellent physical properties owing to microphase separation yielding alternating hard (isocyanate) and soft (long chain polyol) segments $[5,6]$. These features make polyurethanes especially useful as a conformal coating in electronics assemblies besides their versatility in processing, electrical properties, and optical transparency. In addition, efforts in building nanocomposite materials from polyurethanes have been accomplished with relative ease giving way to remarkable increases in their mechanical strength and thermal stability [7]. Previous studies in developing nanoparticle polymer composites have looked at different length scales of filler particles [8] and different inorganic fillers [9-11]. However, in an effort to maintain the viscosity and the transparency of the coatings, nano-sized fillers need to be employed. These filler particles are also expected to contribute to the strength of the matrix due to the composite strengthening effect of the particles in the matrix. Further, in the case of functionalized fillers, as is the case in this study, there is an additional benefit of strong chemical bonding between the particles and the matrix that adds to the overall coating performance. Well functionalized nanoparticles can also reduce the chance for particle agglomeration in the coating.

In this work, isocyanate-functionalized nanoparticles of silica ( $\sim 20 \mathrm{~nm}$ in diameter) in suspension were blended into the polyurethane resin with an expectation to provide mechanical 
improvement. For this, varying amounts of the additive suspensions were blended with the polyurethane base and the resultant composite coatings were characterized to monitor the mechanical properties of the resultant coating. To separate out the extent of the effect of the nanoparticle addition from that of the isocyanate which is included in the particle suspension, another set of sample with only the isocyanate hardener (without nanosilica particles) was also analyzed.

It was also attempted to determine an optimum concentration of nanoparticle addition that would potentially increase tin whisker penetration resistance, which is selected from coating microstructures and mechanical performance. The mechanical properties of the nanocomposite coatings were evaluated using both tensile testing and nanoindentation, the latter of which allows for extremely localized nanoscale mechanical property tests in the 1-2 $\mu \mathrm{m}$ indentation depth range. Infrared spectroscopy was used to characterize various functional groups that are influenced by the coating synthesis and degradation. Low-kV FE-SEM was used to observe the microstructure developments of the nanoparticle-filled polyurethanes and to examine the distribution of the nanoparticles across the coating thickness. These composite coatings should also have the potential to give a better surface coverage on vertical surfaces by adding the nanoparticles to the PU resin that will in turn provide the enhanced whisker mitigation ability.

\section{Experimental Techniques}

\subsection{Materials}

Moisture-curable PU coatings using commercially available Hysol PC18M (Henkel Inc., Irvine, CA) mixed with Desmodur XP2742 suspension (Hexamethylene diisocyanate, HDI, trimer with $20 \mathrm{~nm}$ mean diameter silica nanoparticles) from Bayer MaterialScience (Pittsburgh, PA) were pre-cast in requisite compositions (Table 1). The base polyurethane (100\% PC18M) was used as the control sample and four nanosilica added resins based on weights of the suspension were prepared and cast as coatings. 10\%, 20\%,30\% and 50\% XP2742 suspensions were blended into PC18M to yield each composition containing 3.5\%, 6.74\%, 9.75\% and $15.17 \%$ weight of nanosilica. Further, four more compositions $(5.18 \%, 10.36 \%, 15.54 \%, 25.9 \%)$ 
of Desmodur N3300 (Aliphatic polyisocyanate (HDI trimer) only) from Bayer MaterialScience were added to the PC18M to make the same isocyanate contents as in the XP2742 compositions.

Table 1. List of PU compositions examined in this work.

\begin{tabular}{|c|c|c|c|c|}
\hline $\begin{array}{l}\text { Sample } \\
\text { Number }\end{array}$ & $\begin{array}{c}\text { Base } \\
\text { Polyurethane }\end{array}$ & $\begin{array}{c}\text { wt.\% } \\
\text { suspension } \\
\text { added to base }\end{array}$ & $\begin{array}{l}\text { wt.\% of isocyanate } \\
\text { in suspension } \\
\text { (HDI Trimer) }\end{array}$ & $\begin{array}{c}\text { wt.\% of } \\
\text { nanoparticles }\end{array}$ \\
\hline 1 & PC18M & $0 \%$ & $0 \%$ & $0 \%$ \\
\hline \multicolumn{5}{|c|}{ Nanosilica + Isocyanate(HDI) containing compositions } \\
\hline 2 & PC18M & $10 \%$ XP2742 & $5.18 \%$ & $3.50 \%$ \\
\hline 3 & PC18M & $20 \%$ XP2742 & $10.36 \%$ & $6.74 \%$ \\
\hline 4 & PC18M & $30 \%$ XP2742 & $15.54 \%$ & $9.75 \%$ \\
\hline 5 & PC18M & $50 \%$ XP2742 & $25.90 \%$ & $15.17 \%$ \\
\hline \multicolumn{5}{|c|}{ Isocyanate (HDI)-only containing compositions } \\
\hline $6^{*}$ & PC18M & $5.18 \% \mathrm{~N} 3300$ & $5.18 \%$ & $0 \%$ \\
\hline $7^{*}$ & PC18M & $10.36 \% \mathrm{~N} 3300$ & $10.36 \%$ & $0 \%$ \\
\hline $8^{*}$ & PC18M & $15.54 \% \mathrm{~N} 3300$ & $15.54 \%$ & $0 \%$ \\
\hline $9^{*}$ & PC18M & $25.90 \% \mathrm{~N} 3300-$ & $25.90 \%$ & $0 \%$ \\
\hline
\end{tabular}

('Sample $\# 6, \# 7, \# 8$, and $\# 9$ are a replica of $\# 2, \# 3, \# 4$, and $\# 5$ without nanosilica, respectively)

Nanosilica particles in the XP2742 suspensions are functionalized with an isocyanate (NCO) that forms a bonding between the silica nanoparticles and the polymer matrix when mixed. On the other hand, the N3300 suspension only contains an isocyanate without nanosilica particles. For convenience, in this paper, suspension weights (which includes the solvent, isocyanate, and nanoparticles) of the additives $(10 \%, 20 \%, 30 \%$ and $50 \%)$ will be specified in place of the actual filler weights while referring to the nanosilica samples.

\subsection{Methods}

\subsubsection{Sample Fabrication of Polyurethane Coatings}

All compositions of the coatings tested were prepared by spreading the corresponding resins as thin coatings on glass substrates or slides. $\mathrm{PC} 18 \mathrm{M}$ is a single component polyurethane which cures in the presence of moisture following the evaporation of solvent. In case of PC18M containing the XP2742 (isocyanate + nanosilica) suspension, the presence of the accompanying isocyanate not only bonds the silica to the polyurethane matrix but also increases the hard 
segment concentration in PU. During the pre-processing, the blended resins were cast onto glass substrates with the thickness regulated to be less than $100 \mu \mathrm{m}$ in all cases. The evaporation step was conducted on a hot plate for one hour at $45^{\circ} \mathrm{C}$ followed by curing in an oven regulated at $60 \%$ relative humidity and $60^{\circ} \mathrm{C}$. After curing, the PU coatings were peeled off from the substrates and then used for microstructural observation and tensile testing. For nanoindentation, coatings on the glass slide were directly utilized.

\subsection{Characterization}

\subsubsection{Infrared Spectroscopy (FT-IR)}

Comparative chemical characterizations were performed on peeled coatings by IR spectroscopy (Perkin Elmer Spectrum 100) in an attenuated total reflectance (ATR) mode from the wavenumbers ranging from $650 \mathrm{~cm}^{-1}$ to $4000 \mathrm{~cm}^{-1}$. It was used to assess the relative extent of curing of PU and the change in chemical structures with the addition of various concentrations of the nanosilica and isocyanate to PU base by observing the dominant absorbance peaks.

\subsubsection{Scanning Electron Microscopy (SEM)}

Microstructural analysis of the PU coatings was performed using FE-SEM (Zeiss Supra 55 VP-SEM). Coating surface might not represent for true nature of the PU microstructure due to its restructuring once exposed in air. Therefore, the polyurethane coatings were fractured immediately after freezing in liquid nitrogen to expose their internal microstructure through cross-sectional fracture surface. Furthermore, to avoid electron charging and beam damage, the fractured surfaces were coated with a thin layer of carbon film and viewed in the SEM at a low accelerating voltage $(2 \mathrm{kV})$.

\subsubsection{Nanoindentation}

Depth-sensitive nanoindentation testing was performed using a Hysitron TriboIndenter on PU coatings cast on glass slides. The built-in dynamic mechanical analysis module in the TriboIndenter (called nanoDMA), was used with an increasing load from $500 \mu \mathrm{N}$ to $6500 \mu \mathrm{N}$ coupled with a small added oscillating load at a frequency of $25 \mathrm{~Hz}$. This mode of testing makes 


\section{Results and Discussion}

\subsection{Chemical Analysis}

Figure 1 shows IR spectra with the addition of nanosilica suspension (XP2742) to the polyurethane base. In order to evaluate the relative contribution of each peak, all spectra were normalized by the $\mathrm{C}-\mathrm{O}$ peak that is located at $1064 \mathrm{~cm}^{-1}$. The characteristic urethane peak at $1707 \mathrm{~cm}^{-1}$ decreased in relative intensity when the silica suspension addition changed from $10 \%$ to $50 \%$ in the PU resin. On the other hand, the peak corresponding to the urea bonding (1681 $\mathrm{cm}^{-1}$ ) became more increased as there was an increase in the isocyanate concentration in the coating. The urea formation can be attributed to the reaction of isocyanate with water via the formation of an amine $\left(-\mathrm{NH}_{2} ; 1460 \mathrm{~cm}^{-1}\right)$. The availability of water present during the curing process facilitates this process. Although the primary reaction of the isocyanate is considered to be its reaction with the polyol to produce polyurethane, the high rate of the water-isocyanate reaction can result in urea formation. Further, the $-\mathrm{C}-\mathrm{N}$ bond peak at $1220 \mathrm{~cm}^{-1}$ shows a slight yet distinct decrease with the corresponding increase in the isocyanate (and corresponding nanosilica) content. 

particles to be bound to the polyurethane matrix; consequently, a decreased intensity of the urethane peak is noted with a correspondingly higher intensity of the urea peak as a function of the amount of increased silica suspension. Additionally, an increasingly dominant peak at 1100 $\mathrm{cm}^{-1}$ corresponding to the Si-O bond stretch is also present. This peak is expected to be more intense due to the increased amount of the nanosilica particles and its intensity seems to be directly proportional to the amount of XP2742 added. In the plain polyurethane case (PC18M), the lack of additional isocyanate content restricts the formation of urea by the amine, resulting in a relatively dominant $-\mathrm{C}-\mathrm{N}$ peak.

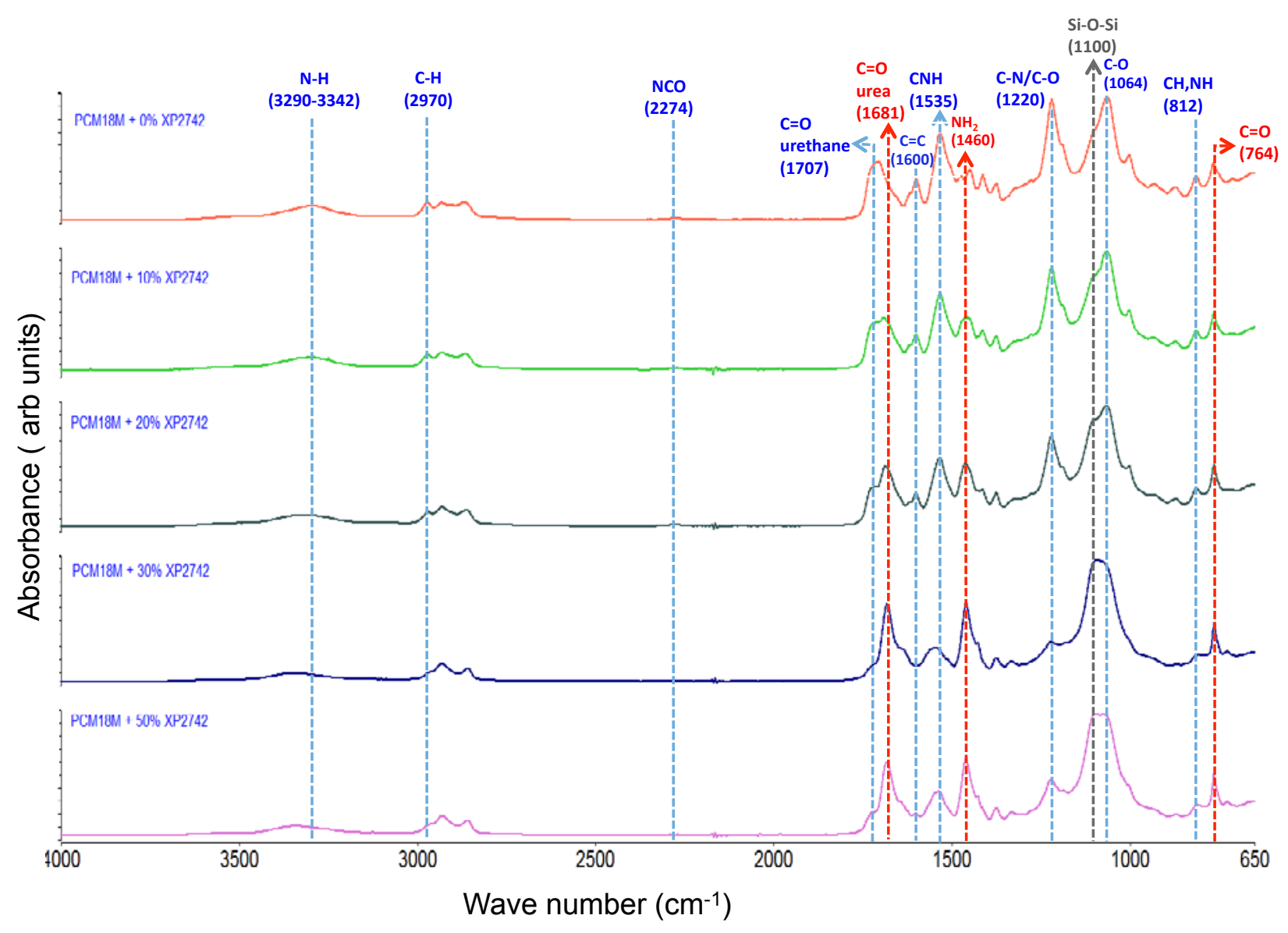

Figure 1. FT-IR spectra of polyurethane with different amount of nanosilica (XP2742) addition. 
There were some similarities with the addition of isocyanate (N3300) only case (figure 2), as seen with an increase in silica suspension concentration in the PC 18M base resin (XP2742). Four different amounts of the isocyanate were compared. It is shown that urea peaks become more dominant with the increased amount of N3300 added. In addition to the two urea related peaks, another peak located at $764 \mathrm{~cm}^{-1}$ is increasing with the amount of the isocyanate. Analysis of these spectra shows clear relations with standard polyurethane chemistry and contains similar critical peaks as noted previously in case of the nanosilica addition case. A small NCO(isocyanate) peak appears at $2274 \mathrm{~cm}^{-1}$ in case of the $5.18 \%$ and $10.36 \%$ samples, which indicates incomplete curing.

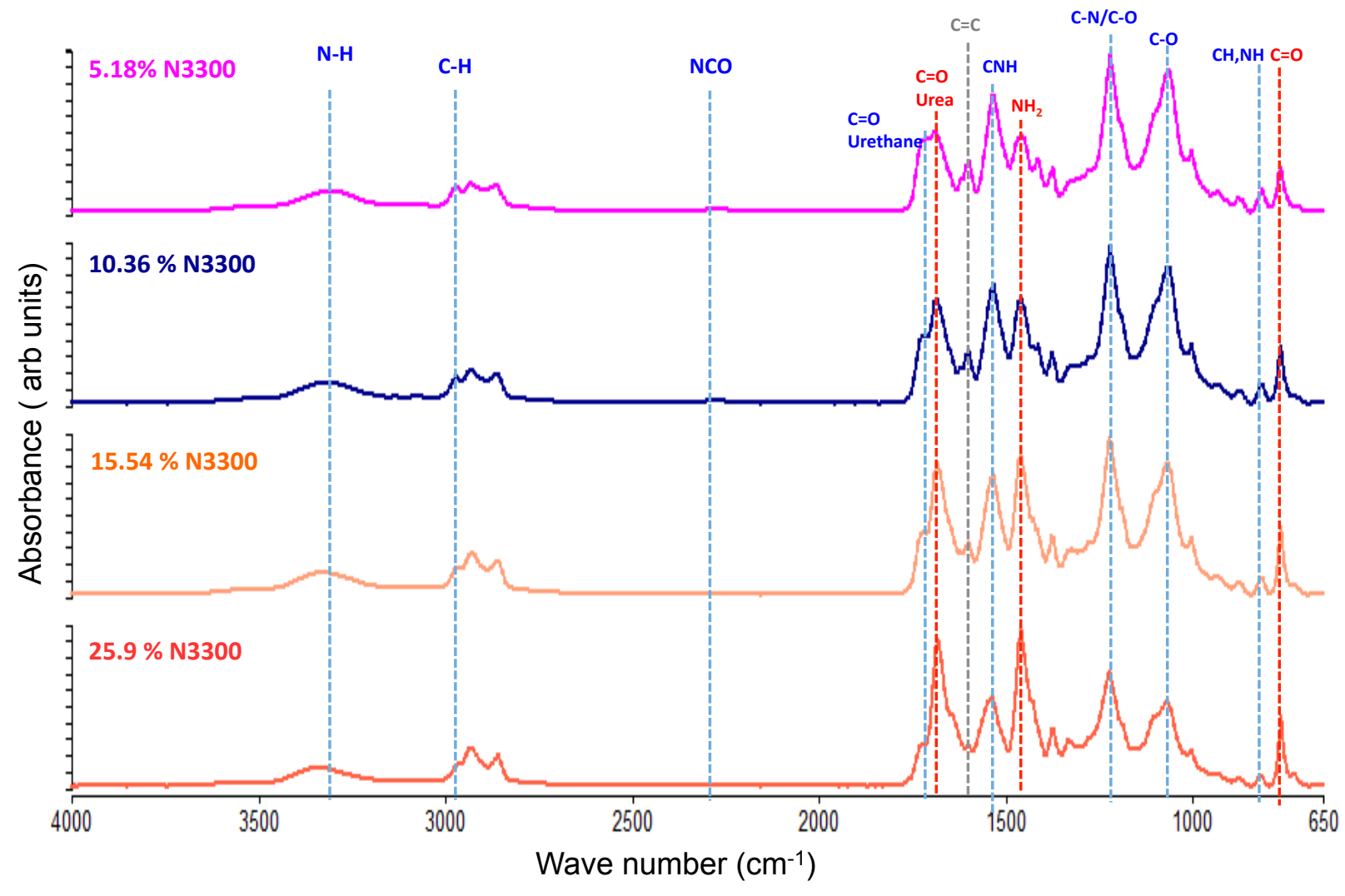

Figure 2. FT-IR spectra of polyurethane with different amount of isocyanate (N3300) addition

\subsection{Microstructures}

Figure 3 shows cross-sectional SEM images of cryo-fractured PU coatings filled with different amounts of nanosilica suspension. For unfilled PU coating, the networked 'wormy' 
structure appears (Fig. 3 (a)). These network structures from the PU were also shown in our previous AFM study [13]. When 20\% nanosilica suspension was added, the microstructure changed to have dimpled domains (i.e., craters) surrounded by the network ridges (Fig. 3 (b)). In this case, nanosilica particles are also clearly visible. These dimpled domains seem to form as a result of the increased isocyanate content (i.e., hard segments) and the domain size decreases with the increasing isocyanate content. It was reported that hard segments within the polymer matrix tend to coalesce and form domains which range from tens to several hundred nanometers in size [12] depending on the type of isocyanate used.

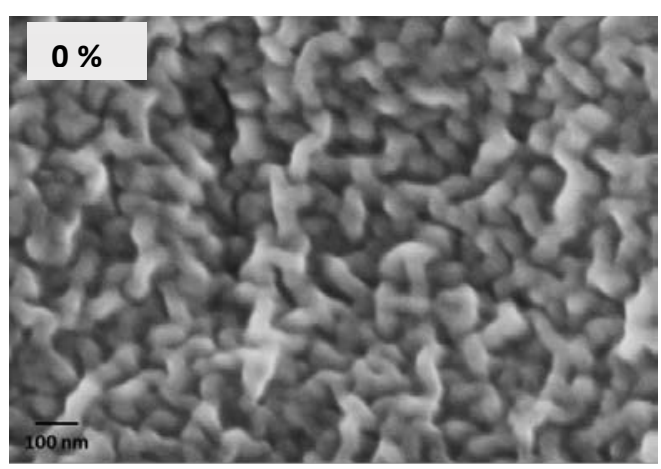

a)

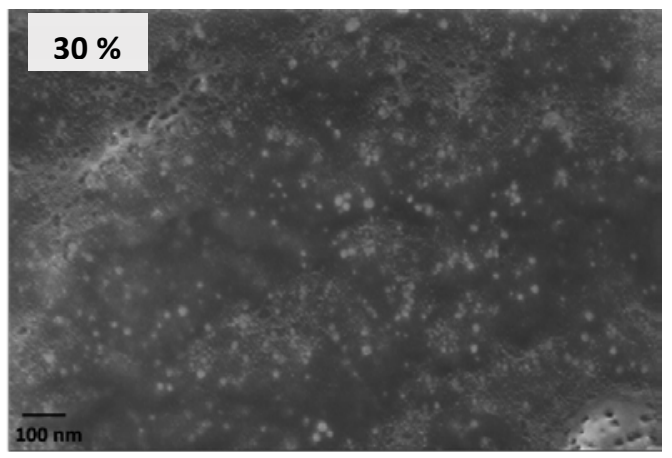

c)

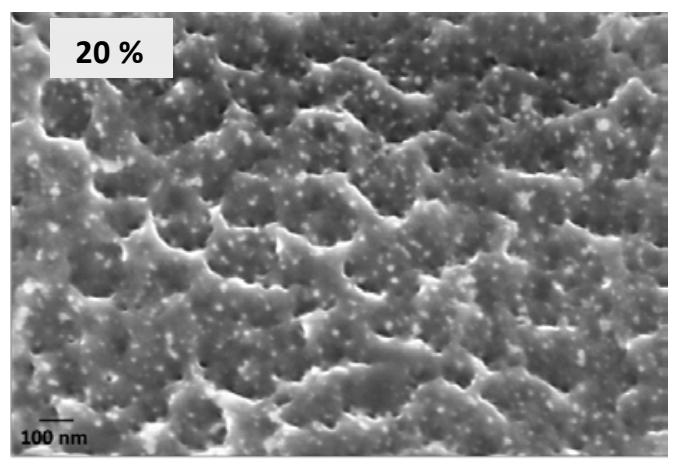

b)

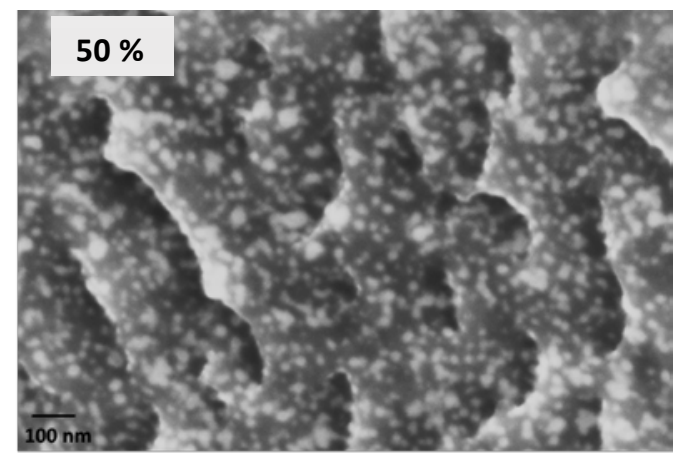

d)

Figure 3. Cross-sectional SEM images of polyurethane with various amounts of nanosilica suspension addition; a) $0 \%$, b) $20 \%$, c) $30 \%$, d) $50 \%$

The relative amounts of the hard and soft domains present in each coating are dependent on the amount of isocyanate hardener added. The increasing amounts of the nanosilica suspension consequently increase the added isocyanate (HDI) (refer to Table 1) leading to higher hard 


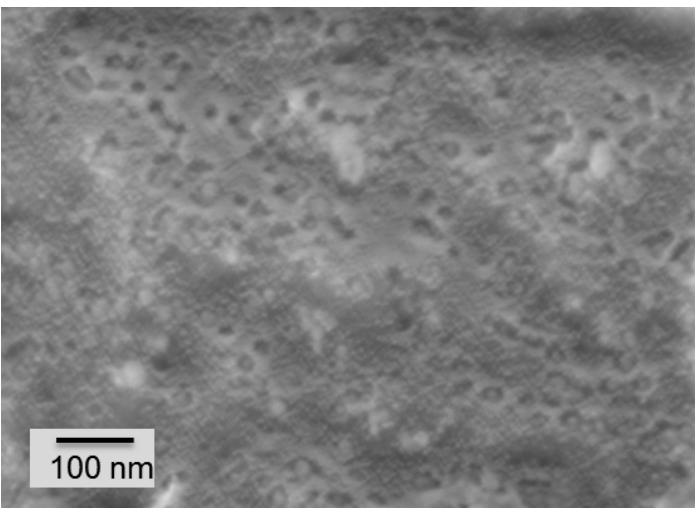

b)

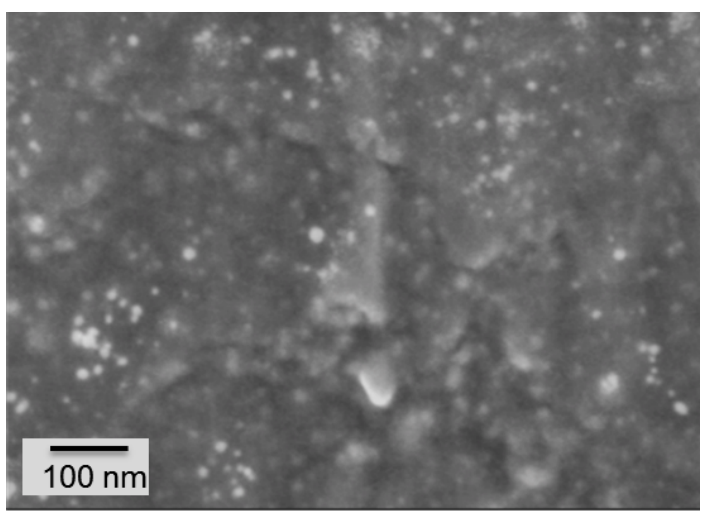

Figure 4. Polyurethane with 30\% nanosilica suspension addition; a) Particle distribution in the

segment concentration in the coating. The aforementioned network structure almost disappeared for the $30 \%$ suspension case although much finer structure could be still found in very localized areas, as seen in Fig. 3 (c). The increased isocyanate concentration in the coatings leads to embrittlement which is demonstrated in the $50 \%$ suspension addition case by nano- and microcracks as shown in Fig. 3 (d). These cracks might have occurred during the sample fracture process, which did not occur for the $30 \%$ addition or lower. This is a consequence of not only the increased isocyanate concentration, resulting in the hard segment increase, but also a result of an increased formation of polyurea in the coating with the increase in the isocyanate content. Furthermore, this polyurea formation generates carbon dioxide gas as a by-product, resulting in inclusions such as gas bubbles in the coating.

The distribution of nanoparticles within the polymer matrix was in general uniform with no evidence of extensive settling. The individual particle size was $\sim 20 \mathrm{~nm}$, which is consistent with the vendor's specification on the suspension. However, some settling began to occur when $30 \%$ suspension or more was added, as shown in Fig. 4. The bottom side of the PU coating clearly shows more nanosilica particles than the top side. Even in this case, particle agglomeration was largely absent. Our TEM studies on the $50 \% \mathrm{SiO}_{2}$ addition case showed singly distributed nanoparticles in the PU matrix [14], which is suggestive of proper surface functionalization of those nanoparticles. This is crucial to make the strong interfacial bonding between the nanoparticles and the matrix that will be of importance in mechanical behavior of the PU.

\author{
top region of the coating, b) Particle distribution in the bottom region
}




\subsection{Nanoindentation of Polyurethane Coatings: 'Localized' Matrix Properties}

Dynamic nanoindentation testing, in an attempt to characterize the mechanical properties of the localized areas of the coatings, was performed on the plain coating (PC18M), along with the four nanosilica added compositions. The motion of the nanoindenter tip can more effectively confine the deformation behavior within PU matrix, but with the influence of the embedded nanoparticles. This will also mimic the penetration behavior of a tin whisker, which will provide direct relevance to tin whisker mitigation. Hardness and elastic modulus versus the indentation depth were plotted for all five samples (Figure 5). Initial hardness variation among all samples ranged from $\sim 180 \mathrm{MPa}$ to $240 \mathrm{MPa}$ until reaching the $400 \mathrm{~nm}$ depth from the top surface of the coating. No clear trend could be observed in this depth range due to the surface factors such as surface roughness and tackiness. However in depth ranges from $500 \mathrm{~nm}$ onwards there is a steady decrease in the hardness with indentation depth for all compositions.

Further, in case of the nanosilica (XP2742 suspension) added coatings, from 10\% to $30 \%$ a dramatic increase in nanohardness was noticed when compared with the plain PC18M coating, followed by a drop in case of the $50 \%$ nanosilica suspension case. While the plain PC18M coating shows a steep gradient of decline and settles at $140 \mathrm{MPa}$ at $1500 \mathrm{~nm}$ depth, the nanosilica-filled coatings range from $170 \mathrm{MPa}$ to $200 \mathrm{MPa}$ at similar depths. A trend similar to the nanohardness was noticed in case of the elastic modulus of the coatings. Up to the $30 \%$ addition, elastic modulus of the nanosilica-filled coatings increased compared to plain PU coating; however, it showed a significant drop at 50\%. Plain polyurethane shows an elastic modulus of around $2.5 \mathrm{GPa}$, while $10 \%$ and $50 \%$ additions increases to $2.7 \mathrm{GPa}$ at maximum depth range with 50\% suspension addition case showing higher data scatter. Further, 20\% and $30 \%$ additions show a similar elastic modulus of $2.8 \mathrm{GPa}$, with greater variation among the $30 \%$ nanosilica addition data points.

The increased polyurea formation can explain the variation in the nanoindentation data in case of the $30 \%$ and $50 \%$ nanosilica addition. It should be noted that the effect of nanoparticles is not the only factor affecting mechanical property data. The effect of isocyanate, which results in increased hard segments and polyurea, is also a critical factor in influencing mechanical performance. Overall, it can be seen that $20 \%-30 \%$ nanosilica addition causes a significant increase in both hardness and elastic modulus in comparison with plain PU. 
Figure 5. (a) Hardness and (b) Elastic Modulus vs. Indentation depth plot for $0 \%$ to $50 \%$ nanosilica suspension in PU coating

As mentioned above, the $50 \%$ suspension case shows a larger variation in nanoindentation data. One possible reason for this large variation could be attributed to the brittleness of this coating as shown in fracture samples. This can be related to the formation of more defects and urea domains in the coatings. Likewise, the $30 \%$ nanosilica suspension coatings showed more 
data scatter than the $20 \%$ counterpart due to more brittle nature although both cases displayed similar property-indentation depth relationships.

\subsection{Tensile Behavior of Polyurethane Coatings: Composite Properties}

Uniaxial tensile testing was performed on polyurethane films containing i) nanosilica+isocyanate (XP2742) and ii) isocyanate only (N3300) to examine a more comprehensive mechanical viewpoint on the effect of the nanoparticles (and isocyanate addition) on the PU coatings. Figure 6 shows representative stress-strain plots for both cases. For all the tests a stress-strain plot was obtained with a clear elastic portion leading to a local stress maximum which is viewed as the yield strength. Total elongation along the gage length of the tensile specimen and the subsequent failure at maximum stress (tensile strength at break) were also compared from these plots.

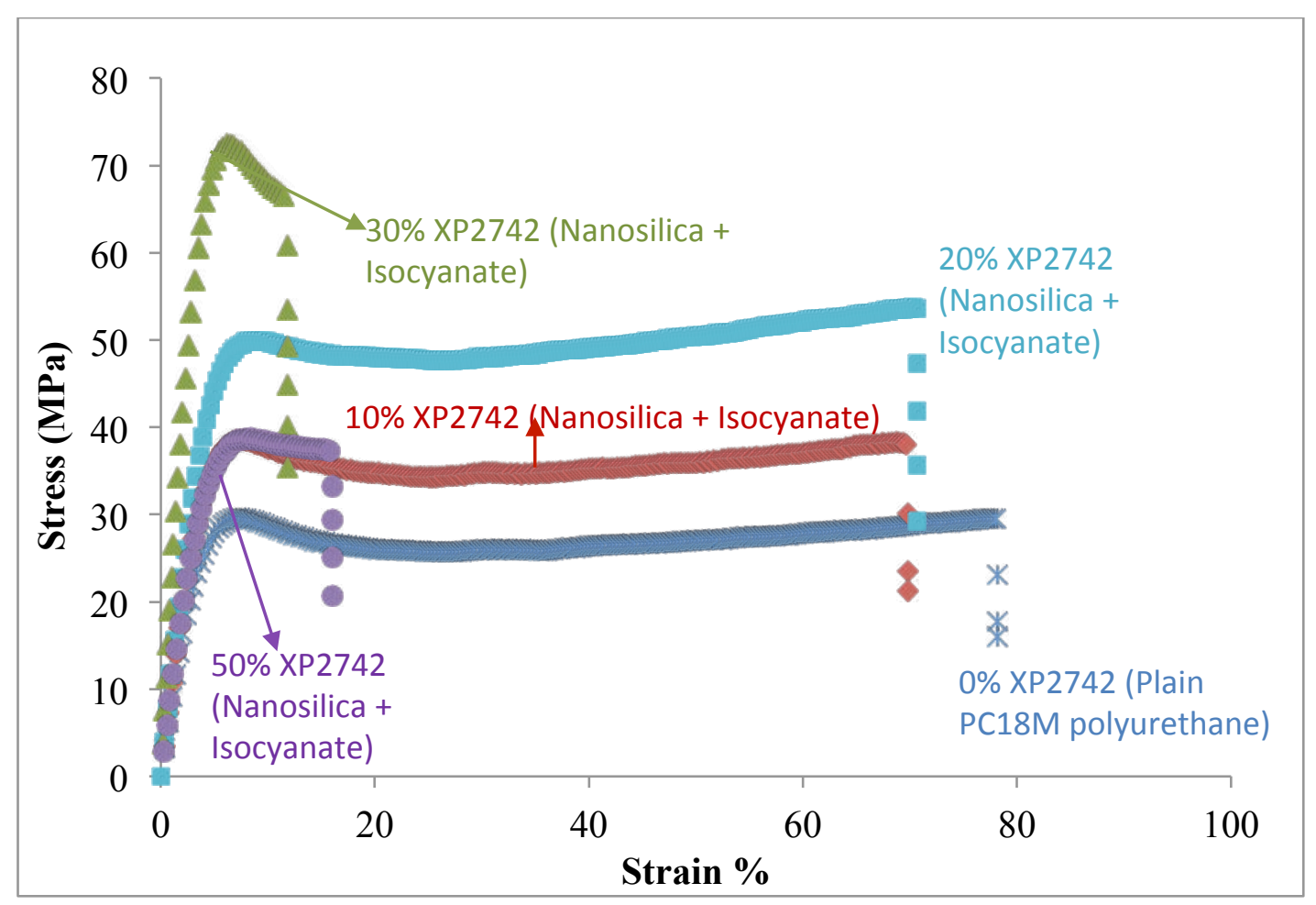

a) 


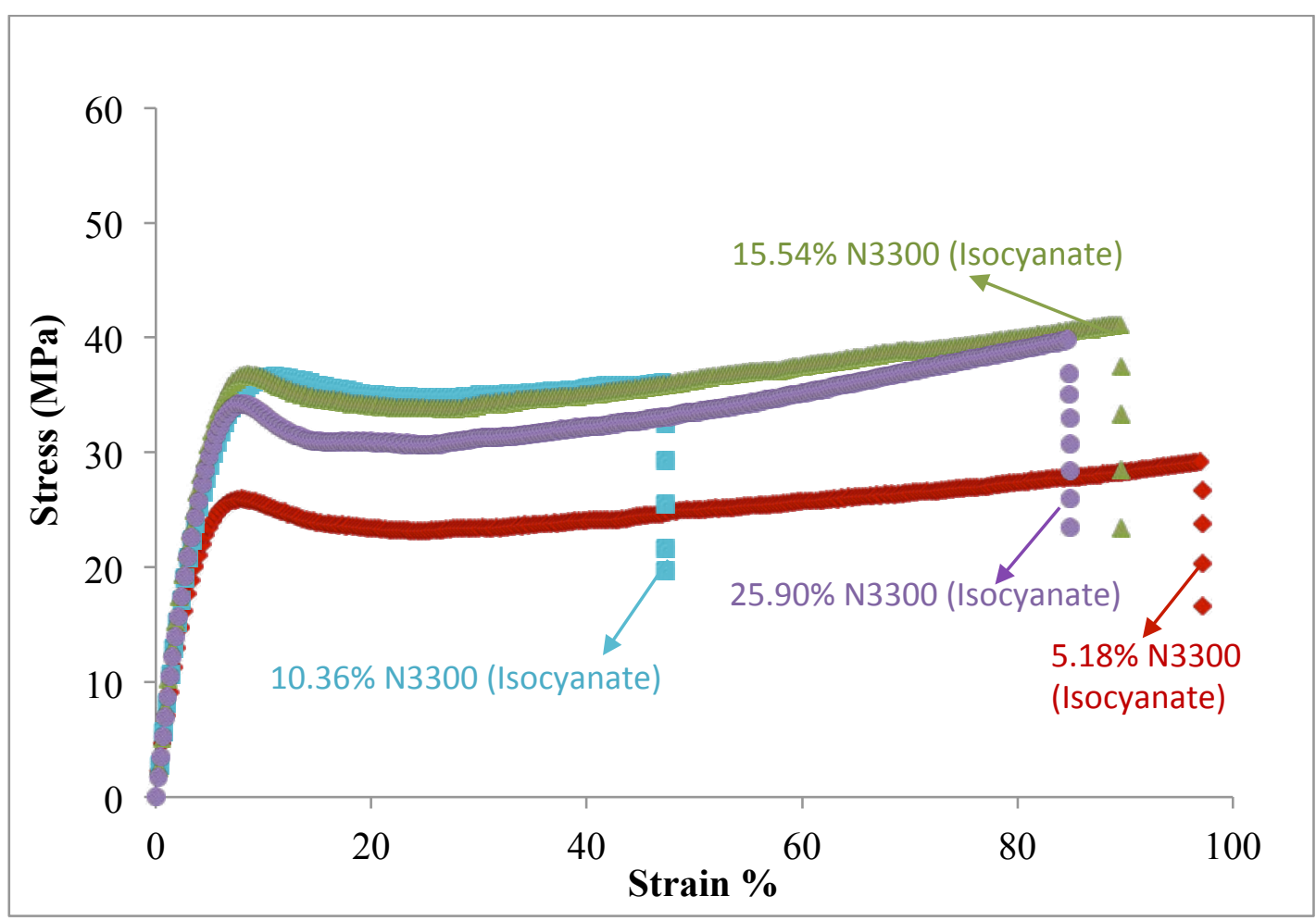

b)

Figure 6. Representative stress-strain plots of - a) Nanosilica suspension addition to $P U$ and b) Isocyanate-only addition to PU

With the addition of nanosilica suspension to the PU coatings, the stress-strain plots are shown in figure 6 a). The non-linear elastic portion of the curve was observed until the yielding point, where necking occurs, followed by its propagation over the gage length during the extended drawing with some strain hardening in all the nanosilica coatings except $30 \%$ and $50 \%$ cases. For $10 \%$ and $20 \%$ cases, both modulus and strength dramatically increase but total elongation was somewhat reduced, compared to those from unfilled PU. As shown in the nanoindentation case, mechanical properties began to degrade after $30 \%$. Especially, the $50 \%$ suspension case showed low strength and brittle behavior, displaying very limited total elongation and large data scattering from sample to sample. A general trend of increasing stiffness and strength in the coatings can be due to the reinforcement provided by the increased hard segments and nanoparticles within the PU matrix. However, as discussed in Section 3.3, mechanical properties of the coatings were critically influenced by the amount of isocyanate which in turn influenced defect population such as voids and urea domains. 
Stress-strain curves of the isocyanate only samples (figure 6b) show a similar trend as in the nanosilica addition. Increased addition of isocyanate results in a slightly steeper elastic region of the stress-strain curve. The yield and tensile strength of the samples also showed a clear improvement until $15.54 \%$, with a decrease in case of the $25.90 \%$ isocyanate addition. As noted in Section 3.2, this could be due to the increased formation of polyurea as well as the concurrent increase in defect population.

In order to differentiate the effect of nanosilica particles from the hard segments, mechanical properties of the PU coatings were compared between those with the addition of nanosilica suspension (i.e., nanosilica + isocyanate) and those with the same isocyanate amount (but no nanosilica particles), as shown in Figure 7. The elastic responses of the polyurethane coatings, both with nanosilica+isocyanate and only isocyanate, showed the similar trend of increasing modulus with an increase in those additions; that is, increasing up to the $30 \%$ suspension case and then decreasing with more suspension, as observed by the elastic modulus chart in Fig. 7 (a). Higher value in the elastic modulus was, however observed when nanosilica particles were added besides the addition of isocyanate. This is indicative of particulate reinforcement provided by the nanosilica, beyond the effect of the hard segments in the polyurethane matrix that restrict chain alignment in the axial stress direction. However, a degradation of the elastic modulus was observed beyond 30\% nanosilica suspension (also, 15.54 $\%$ isocyanate). The drop in elastic modulus is more significant with the nanoparticle suspension case, indicating that the brittleness of PU matrix results in more dramatic impact when combined with the defects resulting from the nanoparticle addition.

This trend of increase and the subsequent decrease in the mechanical property is mirrored in the yield strength and the tensile strength of both sets of samples. Again, a significant increase was observed in the case of nanosilica addition for the yield strength and the tensile strength, which could not be explained by the addition of isocyanate alone (Fig. 7 (b) and 7 (c), respectively). As the hard domains act as a barrier for chain movement and uncoiling following the yield point, the nanoparticles can also suppress the chain motion because the functionalization of the nanoparticles make the strong bonding with the PU network. However, as noted previously (Section 3.1), urea formation in the polyurethane matrix from the reaction of isocyanate in the resin with water proceeds rapidly, and forms regions of urea along with the 
interspersed hard and soft domains. These urea domains become defect sites acting as a stress concentration point that can facilitate premature failure as shown in the $30 \%$ and $50 \%$ suspension cases. In the case of $50 \%$ nanosilica suspension (with $25.90 \%$ isocyanate addition), this mechanism negates the composite strengthening effect of the hard domains and the reinforcement by the nanoparticles, thereby resulting in degradation in the properties.

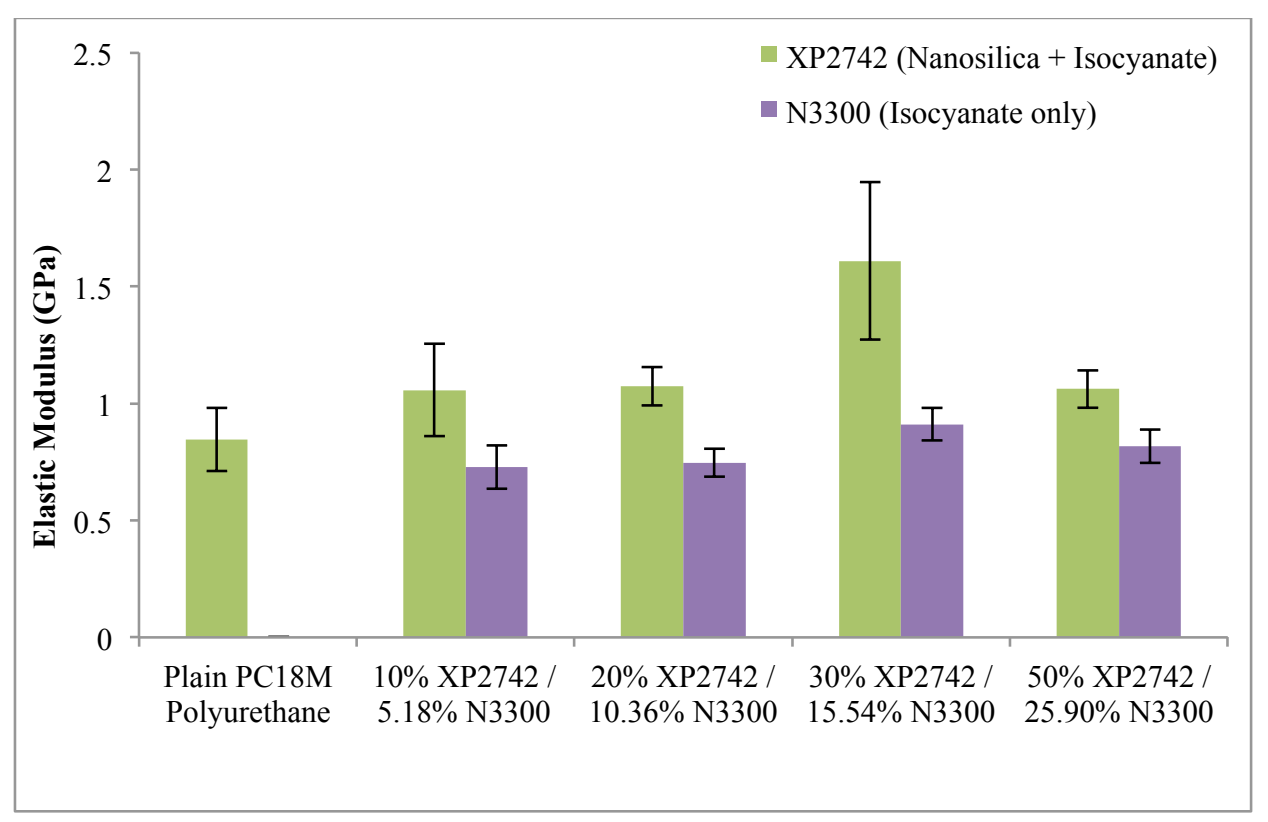

a)

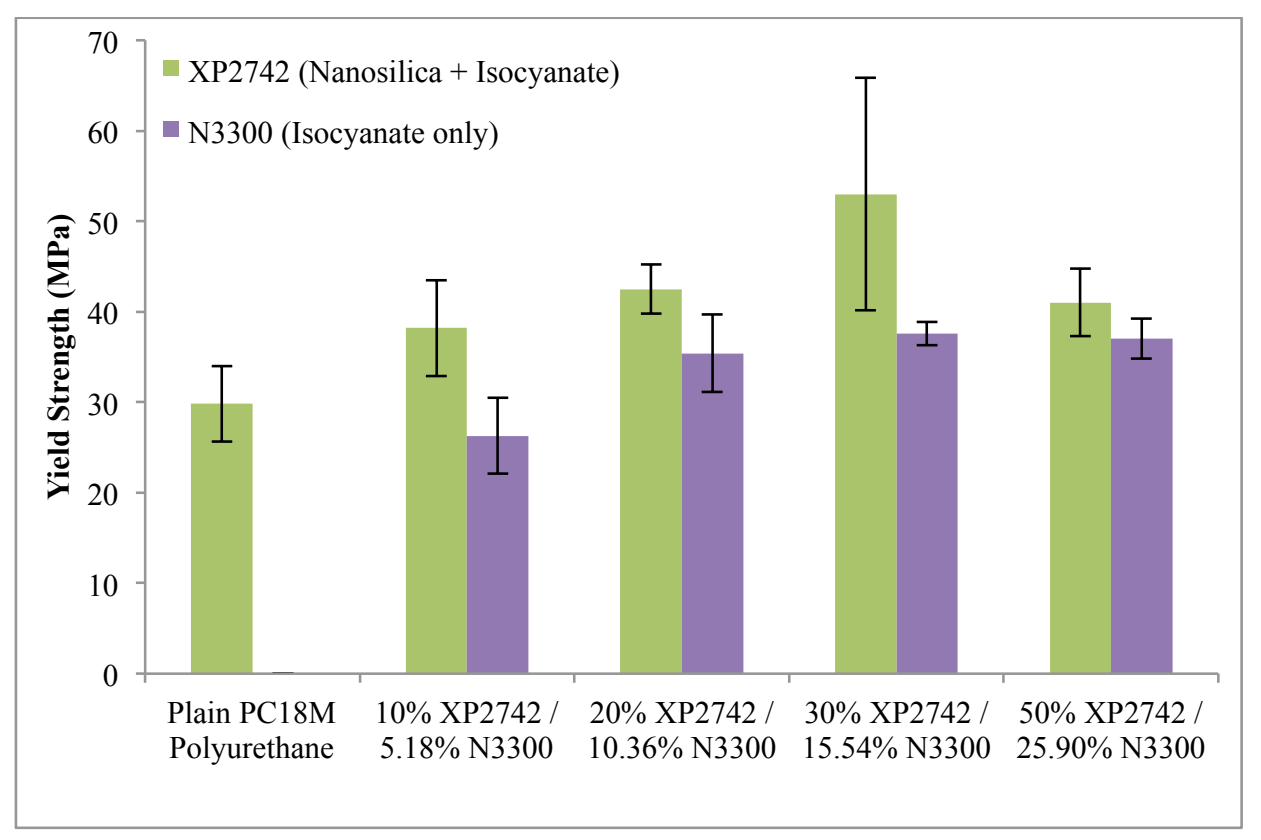

b) 


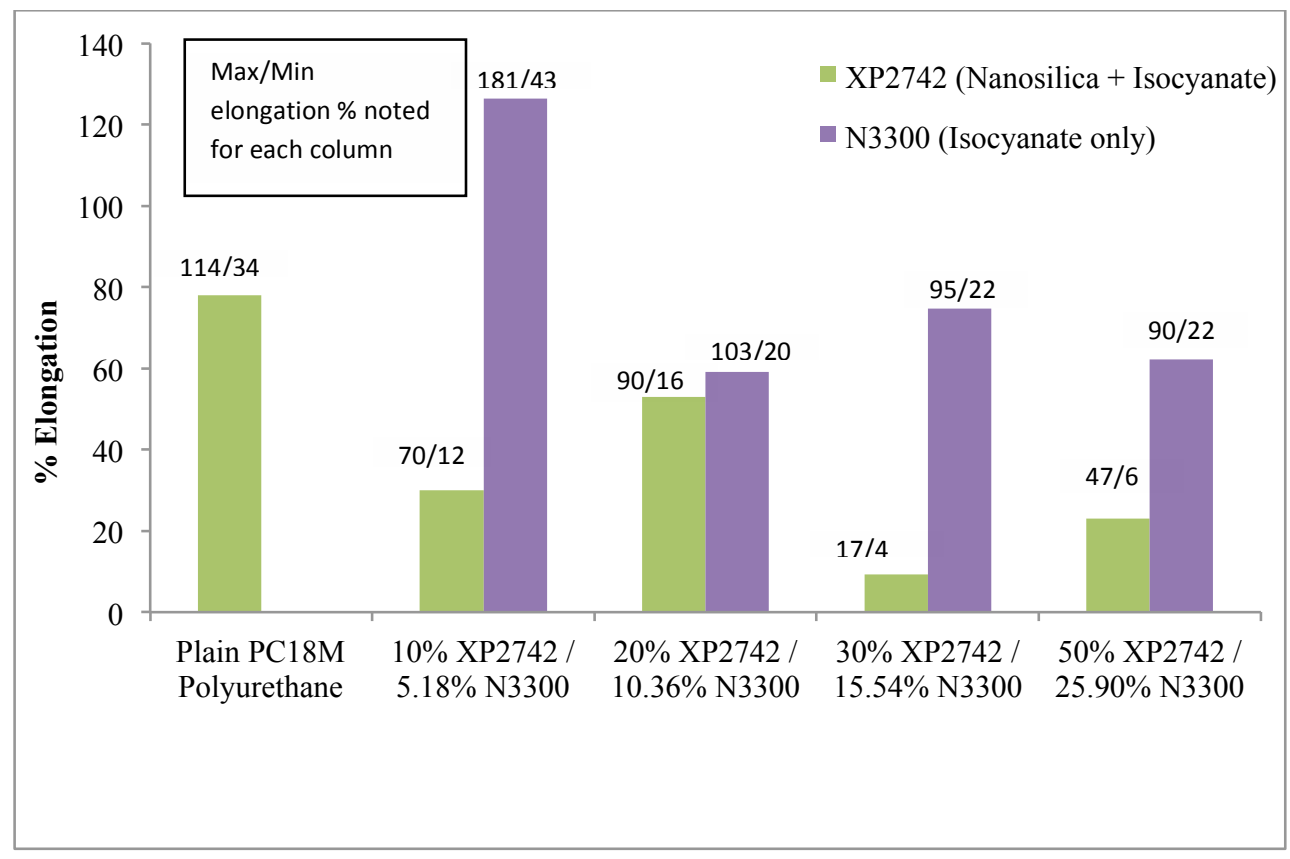

d)

Figure 7. Mechanical properties of nanosilica and isocyanate addition to polyurethane; a) Elastic Modulus, b) Yield Strength, c) Tensile Strength (at break), d) \% elongation 
The $20 \%$ nanosilica addition showed the largest total elongation (ductility) among nanoparticle blended coatings with comparable modulus and strength with the $30 \%$ case. In general, isocyanate only samples display more elongations in comparison. However, the extent of elongation of the coatings showed a significant dependence on sample preparation and defect density in the coatings. Voids, bubbles and other defects served as crack initiators and caused pre-mature failure when the PU matrix became brittle. Figure 8 shows a typical defect (bubble) found in the ruptured coating filled with the $50 \%$ silica suspension that has initiated the crack growth in a brittle manner under tensile loading. Stress concentration at the defect sites was clearly noticeable by bright contrast bands in the C-DIC image.

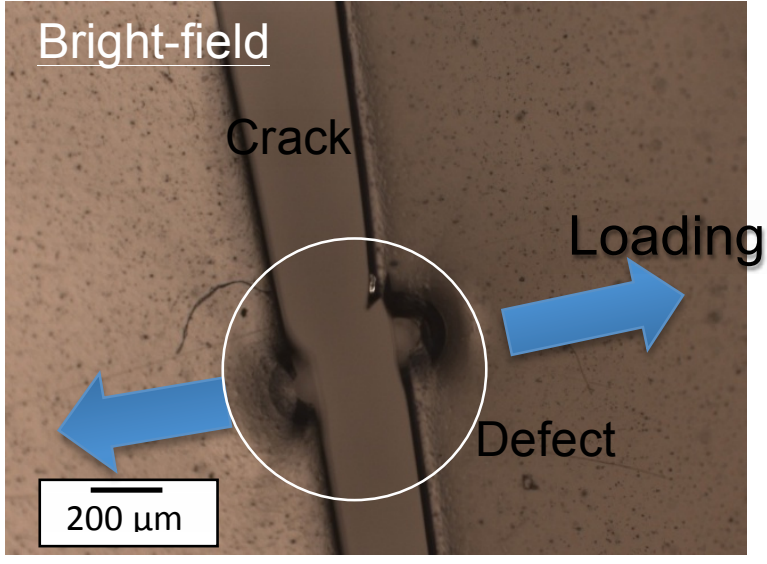

a)

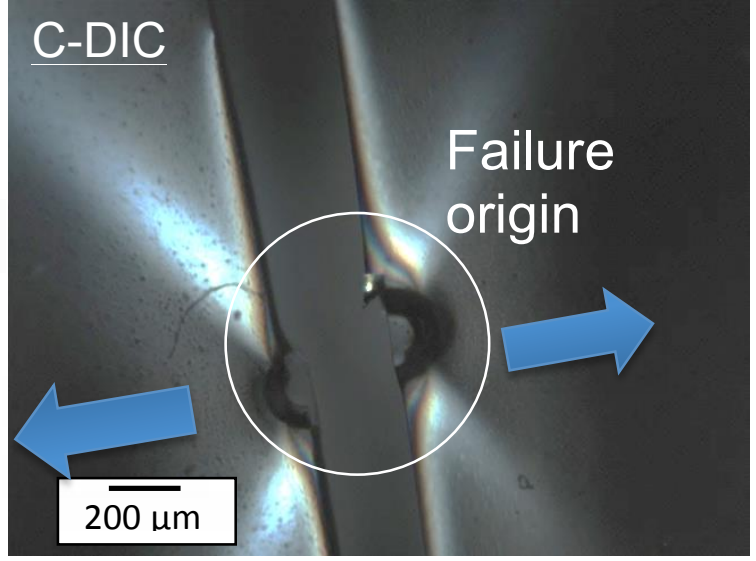

b)

Figure 8. a) Bright-field and b) circular differential interference contrast (C-DIC) image of a defect site in the PU film.

\section{Conclusion}

Nanoparticle-filled PU conformal coatings were analyzed using a combination of highresolution and analytical tools for structural characterization, and nanoindentation and tensile testing for the mechanical behavior of composite structure. IR spectra of the coatings revealed the formation of urea as a by-product during the curing process. With increasing isocyanate content in the particle suspension, a decreased amount of the urethane phase with simultaneous 
increase in the urea phase was observed. Nanoparticle containing coatings showed improved nanohardness, strength, and elastic modulus with 20 wt.\% and 30 wt.\% of nanoparticle suspension (corresponding to $6.74 \mathrm{wt} \%$ and $9.75 \mathrm{wt} . \%$ of nanosilica, respectively) being the most effective in enhancing the mechanical properties of the PU. PU coating with the 30 wt.\% suspension, however, began to display the brittle behavior with limited total elongation. Beyond these concentrations, mechanical properties declined, which was attributed to the degradation of the polyurethane matrix. Microstructural characterization showed the presence of networked 'wormy' patterns and domain structures and these structure features disappeared beyond the 30\% addition. This coincided with the brittleness and premature failure observed in such compositions. The distribution of nanoparticles within the matrix was homogeneous at the $20 \%$ suspension or lower, but it showed the particle settlement after 30\% suspension. There was no significant agglomeration observed even for the 50\% suspension case, which suggests that more particle addition should be possible if the brittleness observed in the PU matrix were prevented.

The formation of increased hard domains and defect densities caused by gas bubbles and polyurea regions in the coating increased the data scattering in the nanohardness and elastic modulus in case of the 30 wt.\% and 50 wt.\% suspension samples $(9.75$ wt. \% and 15.17 wt. \% nanosilica, respectively). Tensile testing showed a dramatic improvement of mechanical properties including elastic modulus, yield strength, and tensile strength with the addition of nanosilica suspension until $30 \mathrm{wt} . \%$ (that contains $9.75 \mathrm{wt}$ \% of silica), with slight improvement also observed in the case of isocyanate only addition up to $15.54 \mathrm{wt}$ \%. Reinforcement provided by the composite strengthening due to the silica nanoparticles was, therefore, chiefly responsible for mechanical property enhancement, whereas excessive urea presence degraded this enhancement at higher nanosilica suspension additions. The $20 \mathrm{wt}$. \% nanosilica suspension (that contains 6.74 wt. \% of silica) showed overall consistency in micro and bulk mechanical properties with relatively less data variation and the most extensive elongation before failure. The plastic deformation behavior of the coatings following the yield point showed a clear dependence on pre-existing defects in the coating with some leading to brittle failure. As a result, it is imperative to restore the ductility and also, the toughness of PU coatings filled with nanoparticles when they are to be used as a conformal coating in electronics assembly to maximize the tin whisker mitigation capability. 


\section{Acknowledgments:}

This work was sponsored by the Strategic Environmental Research and Development Program (SERDP) of the U.S. Department of Defense. Our SEM work was performed at the Analytical and Diagnostics Lab (ADL) in the Small Scale Systems Integration and Packaging ( ${ }^{3}$ IP) Center at Binghamton University. We also acknowledge helpful discussions with and the support from our collaborators from Celestica: Jason Keeping, Polina Snugovsky, Jeff Kennedy, and Marianne Romansky.

\section{$\underline{\text { References }}$}

1) M. Fuse, K. Tsunemi, Cross-Border Impacts of the Restriction of Hazardous Substances: A Perspective Based on Japanese Solders. Environmental Science and Technology 47 (2013) 90289034.

2) W. Fox, L. Woody, Conformal Coatings for Tin Whisker Risk Management. In: Proceedings of IPC APEX Expo 2010, Las Vegas, NV.

3) S. McKeown, J. Kane, S. Meschter, Whisker Penetration into Conformal Coating. In: Proceedings of Proceedings of IPC APEX Expo 2007, Los Angeles, CA.

4) S. Han, M. Osterman, S. Meschter, M. Pecht, Evaluation of Effectiveness of Conformal Coatings as Tin Whisker Mitigation. Journal of Electronic Materials 41 (2012) 2508-2518.

5) S. Huang, J. Lai, Structure-Tensile Properties of Polyurethanes. European Polymer Journal 33 (1997) 1563-1567.

6) L. Xu, P. Soman, J. Runt, C. A. Siedlecki, Characterization of surface microphase structures of poly(urethane urea) biomaterials by nanoscale indentation with AFM. J. Biomater. Sci. Polymer Edn 18 (2007) 353-368.

7) J. H. Li, R. Y. Hong, M. Y. Li, H. Z. Li, Y. Zheng, J. Ding, Effects of ZnO nanoparticles on the mechanical and antibacterial properties of polyurethane coatings. Progress in Organic Coatings 64 (2009) 504-509.

8) Z. S. Petrovic, I. Javni, Polyurethane Elastomers with Nanofillers. Conference Proceedings at ATEC 1998, Atlanta, GA. 
9) J. Zheng, R. Ozisik, R. W. Siegel, Phase separation and mechanical responses of polyurethane nanocomposites. Polymer 47 (2006) 7786-7794.

10) Z. S. Petrovic, J. Y. Cho, I. Javni, S. Magonov, N. Yerina, D. W. Schaefer, J. Ilavsky, A. Waddon, Effect of silica nanoparticles on morphology of segmented polyurethanes. Polymer 45 (2004) 4285.

11) B. Xu, Y. Q. Fu, W. M. Huang, Y. T. Pei, Z. G. Chen, J. T. M. De Hosson, A. Kraft, R. L. Reuben, Thermal-Mechanical Properties of Polyurethane-Clay Shape Memory Polymer Nanocomposites. Polymers 2 (2010) 31-39.

12) C. Prisacariu, Polyurethane Elastomers, Springer-Verlag/Wien, New York, 2011.

13) J. Cho, S. Meschter, S. Maganty, D. Starkey, M. Gomez, D. Edwards, A. Ekin, K. Elsken, J. Keeping, P. Snugovsky, J. Kennedy, Characterization of Hybrid Conformal Coatings Used for Mitigating Tin Whisker Growth. Conference Proceedings at the International Conference on Soldering \& Reliability, Toronto, Canada, 2013.

14) J. Cho, S. Meschter, S. Maganty, D. Starkey, M. Gomez, D. Edwards, A. Ekin, K. Elsken, J. Keeping, P. Snugovsky, J. Kennedy, M. Romansky, Polyurethane conformal coatings filled with hard nanoparticles for tin whisker mitigation. Journal of SMT 27 (2014) 15-21. 